Hanspeter Marti

\title{
Disputation und Dissertation in der Frühen Neuzeit und im 19. Jahrhundert - Gegenstand der Wissenschaftssprachgeschichte?
}

\section{Einleitung}

Wie weithin bekannt, wurde 1753 unter dem Vorsitz des Greifswalder Logikund Metaphysikprofessors Peter Ahlwardt eine deutschsprachige Dissertation über den Nutzen von in deutscher Sprache verfassten Thesenschriften verteidigt (Marti 1998: 59f.; Alvermann 2007: 91; Döring 2007: 137 f.; vgl. auch den Beitrag von Alvermann in diesem Band). Der Gegenstand und die Art seiner Behandlung (auf deutsch) stellten ein Novum in der Geschichte des frühneuzeitlichen Disputationswesens dar. Der Greifswalder Gelehrte setzte sein Postulat in weiteren deutschsprachigen Dissertationen in die universitäre Praxis um (Marti 1998: 61). Unter den Zehntausenden von Disputationsschriften, die in der Frühen Neuzeit in deutschsprachigen Ländern gedruckt wurden, waren aber keine fünfzig in deutscher Sprache verfasst. Christian Thomasius, der gefeierte Pionier der deutschen Wissenschaftssprache, hielt sich, was meist vergessen wird, in allen unter seinem Vorsitz abgehaltenen Disputationen strikt an die traditionelle Gelehrtensprache, das Latein (Marti 2005: 324). Dissertationen waren an Universitäten deutschsprachiger Länder noch in der ersten Hälfte des 19. Jahrhunderts fast ausnahmslos, meist auch noch weit später, in Latein geschrieben (Marti 1998). An der Berliner Universität gab es bis 1866 nur Dissertationen in lateinischer Sprache. Versteht man also unter ,Früher Neuzeit' die Epoche, die um zirka 1800 endete, und fasst die bis dahin entwickelte Textgattung ,Dissertation“ ins Auge, bietet sich dem Wissenschaftshistoriker ein eindeutiges Bild dar: Latein war das Medium gelehrter Kommunikation.

Ewald Horn, der Ende des 19. Jahrhunderts den frühneuzeitlichen Dissertationen eine heute noch gerne herangezogene Monographie widmete, hatte $\mathrm{zu}$ seinem Gegenstand ein ambivalentes Verhältnis: Einerseits bereiteten ihm als Bibliothekar die große Mehrzahl der Massenprodukte wissenschaftlicher Arbeit

Hanspeter Marti, Arbeitsstelle für kulturwissenschaftliche Forschungen, Sernftalstr. 77, CH-8765 Engi, E-Mail: marti-weissenbach@forschungen-engi.ch

Ә Open Access. (c) 2018 Hanspeter Marti, publiziert von De Gruyter. (c) BY-NC-ND Dieses Werk ist lizenziert unter der Creative Commons Attribution-NonCommercial-NoDerivatives 4.0 Lizenz. 
und das tiefe Niveau dieser Universitätsschriften auch wegen Platzmangels in den Bibliotheken Sorgen, und er wollte die Doktordissertationen sogar abgeschafft wissen (Horn 1893: 119f.), andererseits entriss er die damals seit Jahrzehnten unbeachtet gebliebenen frühneuzeitlichen akademischen Kleinschriften dem Vergessen und verlieh ihnen, vielleicht nolens volens, langfristig wissensgeschichtliche Aktualität. Das 19. Jahrhundert brachte innerhalb der philosophischen Fakultät, die damals sowohl naturwissenschaftliche als auch philosophisch-historische Fächer umfasste, eine früher nicht gekannte Diversität wissenschaftlicher Disziplinen hervor, zu denen auch die Geschichte der mittelalterlichen Literatur und der deutschen Sprache zählten. Unter der Rubrik ,Philologie, Archäologie und Sprachwissenschaft“ bot zum Beispiel die Universität Greifswald im Wintersemester 1874/75 Übungen zur deutschen Sprache und Literatur, über die Nibelungen, weitere Lehrveranstaltungen zur älteren deutschen Literaturgeschichte, Übungen zum Mittelhochdeutschen sowie eine Lehrveranstaltung zur deutschen Grammatik, ferner unter der Leitung eines Altphilologen immer noch Disputierübungen an (Verzeichniß der Vorlesungen 1874: 7). Spezielle Themen zur deutschen Sprachgeschichte wurden an vielen Universitäten deutschsprachiger Länder nicht nur in Vorlesungen, sondern auch in Dissertationen mit dem Anspruch, einen Forschungsbeitrag zu leisten, behandelt. So gesehen sind die Dissertationen, vor allem seit dem 19. Jahrhundert, zweifellos maßgebliche Quellen der Wissens-, Wissenschaftsund Forschungsgeschichte.

Im Folgenden nähere ich mich der Titelfrage, indem ich von der frühneuzeitlichen disputatio und Einzelaspekten der Sprachwahlthematik ausgehe und in Bezug auf das 19. Jahrhundert die Universitäten Berlin und Breslau genauer betrachte. Das Rahmenthema erschien im Hinblick auf meinen Gegenstand zunächst äußerst sperrig und daher ungeeignet. Darf und soll man den Begriff ,Wissenschaft' überhaupt auf die Frühe Neuzeit, mehr noch auf die von etwa 1500 bis 1800 entstandenen Dissertationen anwenden? Der kurze historische Exkurs, den ich hier vorlege, führt von einem gattungsgeschichtlichen Einzelaspekt auf grundsätzliche Fragen der Wissenschaftssprachthematik.

In der Frühen Neuzeit wurde aus verschiedenem Anlass disputiert (Marti 1994a/b; Marti 2011): in Privatkollegien übungshalber, übungshalber auch öffentlich, meist auf der Basis gedruckter Dissertationen (exercitii causa), öffentlich beim Verlassen einer Hohen Schule (dissertatio valedictoria), öffentlich während des Studiums zum Zweck des Leistungsnachweises, vor allem von Stipendiaten, ferner pro loco, das heißt im Wettbewerb um eine Stelle an der Universität (Marti 2014b), stets öffentlich zur Erlangung eines akademischen Grades. Aus den Titelblättern der Dissertationen und/oder aus den in sie integrierten Paratexten, den Widmungen und Gratulationen, geht oft der Grund 
der disputatio hervor, zu der mit der Thesenschrift offiziell eingeladen wurde. Von den verschiedenen Dissertationsarten der Frühen Neuzeit blieben nur die Inauguraldissertation und die Habilitationsschrift übrig. Gedruckte Übungsdissertationen wurden in der zweiten Hälfte des 18. Jahrhunderts sehr selten, und mit ihnen verschwanden, von einigen Ausnahmen abgesehen, die an akademischen Gymnasien und anderen nichtuniversitären Hohen Schulen verteidigten Thesendrucke (Marti 2001).

Frühneuzeitliche Dissertationen konnten entweder vom Präses, der die Disputation leitete, oder vom Respondenten, der die Thesenschrift verteidigte, geschrieben sein. Hin und wieder verfassten sogar - in der Regel anonym bleibende - Drittpersonen die Dissertationen. Meistens waren es Gemeinschaftsarbeiten, die in enger Kollaboration von Präses und Respondent entstanden. Selbst den Vermerken ,auctor-respondens ${ }^{\star}$ auf den Titelblättern ist, wenn sie als einziges Identifikationsmerkmal der Verfasserschaft angeführt werden, nicht ganz zu trauen. Gemeinsam haben die späteren Dissertationen mit den frühneuzeitlichen die Themenvielfalt, was sie für fast alle Fragestellungen zu wichtigen, jedoch lange Zeit weit unterschätzten wissensgeschichtlichen Quellen macht.

Vor allem in der Frühen Neuzeit war die Sprache nicht nur ein Thema von Dissertationen, die unter dem Patronat der philosophischen Fakultäten entstanden (Beispiel aus der juristischen Fakultät der Universität Halle: Ludovici \& Leyssner 1720). Zwar sind die einschlägigen philosophischen Thesendrucke deutscher Länder der Zeit von 1660 bis 1750, von einzelnen Hohen Schulen abgesehen, bibliographisch zu einem großen Teil erfasst (Marti 1982), bislang aber nicht im Blick auf die Sprachenfrage systematisch untersucht, mit anderen Gattungen gelehrter Literatur verglichen und den historischen Kontexten zugeordnet worden.

\section{Das sprachtheoretische Spektrum am Beispiel ausgewählter frühneuzeitlicher Basler Dissertationen}

In den Jahren 1688 bis 1692 ließ der Oratorikprofessor Samuel Werenfels (16571740; Biographie: Marti-Weissenbach 2017: 228-243) in lockerer Abhängigkeit von René Descartes sieben Dissertationen über die Logomachie, den gelehrten Streit um bloße Worte, verteidigen (Rother 2003; Rother 2007). Da sie das ganze 18. Jahrhundert hindurch, zu einem einzigen Traktat vereinigt, in Deutsch- 
land, der Schweiz und den Niederlanden in Sammeleditionen werenfelsscher Werke gedruckt wurden, fanden sie weite Verbreitung (zu den Ausgaben: Marti-Weissenbach 2017: 229 f.; Werenfels 1739: 1-116). ${ }^{1}$ Der Basler Verfasser enthielt sich in diesen Thesenschriften historischer Spekulationen, zum Beispiel über den Ursprung der Sprachen und über ihren Rang. Er beschäftigte sich auch nicht mit den Eigenschaften bestimmter Sprachen und deren Eignung für die gelehrte Kommunikation, sondern mit sprachtheoretischen Fragen, genauer mit den logisch-rhetorischen Anforderungen, die zu erfüllen seien. An erster Stelle der für natürliche Sprachen universalen Kriterien stehen für Werenfels, der die Rhetorik logikzentriert bestimmt, die Klarheit respektive Deutlichkeit (perspicuitas) und die Nachvollziehbarkeit der Aussage, wie sie in der Mathematik erreicht werde (Werenfels 1739: 113). ${ }^{2}$ Ferner finden die zur selben Zeit in Christian Thomasius' Vernunftlehren akzentuierte Vorurteilskritik sowie eine die menschlichen Affekte und die Selbsterforschung betreffende, heute bisweilen Kardiognostik genannte Psychologie Beachtung (Werenfels 1739: 114). ${ }^{3}$ Die Gelehrten sollen sich an den allgemeinen Wortgebrauch halten, neue Wörter nur im Notfall einführen, sie kennzeichnen, wenn ihre Verwendung unumgänglich sei (Werenfels 1739: 113). ${ }^{4}$ Logomachieverdächtige Sachwörter müssten in einem Universallexikon in alphabetischer Reihenfolge samt ihren semantischen Implikationen mit Beispielen festgehalten werden (Weren-

1 Hier wird die letzte von Samuel Werenfels durchgesehene, um weitere Texte vermehrte Ausgabe von 1739 zu Grunde gelegt; bei im engen Sinn disputationsgeschichtlichen Studien ist auf die Erstauflage, die Dissertationen, die zu den Disputationen einluden, zurückzugreifen. Dort finden sich auch die Namen der Respondenten und weitere anlassbezogene Informationen.

2 Zur perspicuitas: „Accedat perspicuitas, quanta potest maxima. [...] Persuade tibi, posse aliquem nimis ornate, nimis acute, nimis sublimiter, nimis copiose, nimis presse, nimis venuste loqui: nimis perspicue docentem loqui non posse.“

3 Vorurteilslosigkeit beim Lesen: „[...] sed vel praecipue si animum ad lectionem afferimus vacuum ab affectibus pravis et praejudiciis, quae nos invitos à verborum sensu saepissime abducunt.“ Den schädlichen Affekten wird als erkenntnisfördernd die Liebe zur Wahrheit (,amor veritatis“) entgegengestellt.

4 Allgemeinverständlichkeit: „Cogita, te scribere non doctis modo, sed rudibus, non ingeniosis modo, sed stupidis; non hoc tantum, sed aliud agentibus; non vacuis tantum, sed occupatis, non attentis modo, sed volaticis ingeniis; non amicis modo, sed aemulis; non bonis tantum interpretibus, sed malitiosis etiam calumniatoribus [...]“. Kennzeichnung: „Si qui coguntur multis in libro aliquo verbis uti ambiguis, aut consuetas vocabulorum significationes mutare; multas hi Logomachias praecavebunt, si libro indicem horum verborum adjungent cum accuratissimis singulorum definitionibus; in ipso autem libro haec verba à caeteris aut charactere diverso, aut alia nota distinguent.“ 
fels 1739: 94 f.). ${ }^{5}$ Vorbild für den Sprachgebrauch der Gelehrten ist für Werenfels eine von Unklarheiten und Ambiguitäten gereinigte ideale Umgangssprache und letztlich die mathematische Definition, die auch außerhalb der Mathematik verwendet werden könne (Werenfels 1739: 114). ${ }^{6}$ Entsprach eine Aussage den definitorischen Anforderungen, die im Prinzip jedes Idiom erfüllen konnte, stand für Werenfels der Verwendung einer beliebigen Sprache für gelehrte Zwecke nichts im Weg. Hätten sich die Gelehrten an die von ihm vorgeschlagene Kennzeichnung und Erklärung unüblicher Termini gehalten, hätte hieraus die künftige sprachhistorische Forschung empirischen Nutzen ziehen können, aber wohl auch eine sprachliche Verarmung feststellen müssen.

Das Latein genügte freilich den skizzierten Normvorschriften, und es bedurfte nicht der Ausbildung von Kunstsprachen, wie sprachtheoretische Entwürfe der Frühen Neuzeit (Eco 1994), häufig mit biblizistisch-theologischen Argumenten, z. B. der Instrumentalisierung des Sündenfalls und des Turmbaus von Babel (Borst 1995), sie vorsahen. Der Basler Oratorikprofessor vertrat einen typisch frühaufklärerischen Denkstil (zum Terminus ,Denkstil‘: Fleck 1980; Schiewe 1996) und war ein Vorläufer der philosophischen bzw. logikzentrierten Oratorie, die später der an verschiedenen Gymnasien unterrichtende Johann Andreas Fabricius (1696-1769) und der Jenaer Professor Friedrich Andreas Hallbauer (1692-1750) propagierten (Grimm 1983; Beetz 1980; Till 2004: 340360). In der 1694 verteidigten Dissertation De meteoris orationis, die von Johann Christoph Gottsched (1700-1766) zustimmend aufgenommen und im Gottschedkreis sogar ins Deutsche übersetzt wurde (Marti 2016a; Marti-Weissenbach 2017), lehnte Werenfels barocken Schwulst entschieden ab.

Einen anderen Zugang zur Sprache der Gelehrten fand ein halbes Jahrhundert später Johann Bernhard Merian (1723-1807), der an der Universität Basel

5 Über das Bedeutungs-Wörterbuch, für das ein erfahrener Gelehrter oder vielmehr, da die Arbeit einen Einzelnen überforderte, eine Gruppe kompetenter Mitarbeiter zuständig sein soll, entwickelte Werenfels eine klare Vorstellung: ,ubi omnes voces et loquendi formae, quaecunque in hoc usque tempus Logomachiarum in scientiis et disciplinis omnis generis caussae fuerunt, secundum literarum seriem explicarentur: atque ita quidem, ut nota aliqua à caeteris distinguerentur voces et locutiones nullius significationis; voces significationis incertae; voces significationis inconstantis apud eosdem auctores; voces significationis confusae: in reliquis, quae varias quidem apud varios, sed certas, constantes et distinctas notiones significant, omnes accurate enumerarentur; ad mentem auctorum perspicue definirentur, additis, ut in Lexicis fit, ad singulas definitiones auctorum exemplis.“

6 „ubi [im Wörterbuch] voces eruditis usitatae unico modo omnes clarissime definirentur: sique adigi possent aliqua ratione eruditi, ut in hac vocum acceptione omnes convenirent. Hoc fieri posse, docent Mathematici: quod si enim illi in significatione verborum sibi propriorum accuratissime consentiunt, quare idem in aliis disciplinis sperari non possit, non video.“ 
mehrmals vergeblich eine Professorenstelle anstrebte und dann wie manche seiner Landsleute im Ausland Karriere machte (Marti 2016a). Als Mitglied der Preußischen Akademie der Wissenschaften vertauschte er in seinen Abhandlungen das Latein mit dem Französischen und wurde damit dem anlässlich seiner Bewerbung um die Professur für Oratorik formulierten Postulat untreu, dass die traditionelle Gelehrtensprache beizubehalten und zu fördern sei.

Merians pro cathedra-Dissertation, die Cogitationes quaedam de contemptu linguae Latinae von 1743, entstand in einer Zeit, in der das Latein unter starken Legitimationsdruck geriet und akademischer Fürsprache bedurfte. In Halle und in Göttingen, an den beiden Aufklärungsuniversitäten, widersetzten sich verschiedene Gelehrte der damals starken Tendenz, das Deutsche in der gelehrten Kommunikation zu verwenden. An der halleschen Fridericiana stand der Rhetorik- und Geschichtsprofessor Christoph Cellarius (1638-1707), in Göttingen der Neuhumanist Johann Matthias Gesner (1691-1761) für eine Verbesserung des Lateinunterrichts ein, und beide versuchten dem Latein weiterhin universitären Rückhalt und Ansehen zu verschaffen (Paulsen 1896: 531-534; Beims 2015: 301-357; Marti 2017). In der Topik vielfältiger zeitkritischer Einwände, die Merian gegen nicht namentlich genannte Widersacher vorbrachte, spiegelt sich die damalige Kontroverse um die Eigenart und die Eignung einer bestimmten Sprache, des Lateins, als einziger Gelehrtensprache. Die Thesenschrift nimmt im weiten Feld des Vorzugsstreits eine mittlere Position ein, wie sie sich vor allem in der Vorurteils- und Pedantismuskritik der thomasianischen Vernunftlehren findet. Auch bei Merian betraf Letztere das in eine Auxiliarfunktion abgedrängte Handwerk des Philologen. Dessen Berechtigung zweifelte Merian keineswegs an, aber es stellte für ihn nur eine Vorstufe zu der mit der Philosophie identifizierten und moralischen Ansprüchen genügenden eigentlichen Gelehrsamkeit dar (Merian \& Burckhardt 1743: 10 f.). ${ }^{7}$ Die verba ordnete er zwar mit der erwähnten frühaufklärerischen Logikzentriertheit übereinstimmend den res nach, gleichzeitig ließ er aber die maßvolle rhetorisch-ästhetische Formung der Aussagen zu. Merian distanzierte sich, wie angedeutet, zwar vom praeiudicium novitatis, noch mehr aber vom praeiudicium antiquitatis und da-

7 „Cum vero beatitudo nostra in praxi Virtutis, ad quam per cognitionem Veritatis devenitur, sita sit, manifestum est eo ipso, Linguarum culturam, Artemque Criticam non esse ultimum nostrorum finem, seu quod idem est, non esse veram Eruditionem; Ibi enim non quaeritur, quid verum sit? quid falsum? sed duntaxat, quomodo Autores, sive vera illi scripserint, sive falsa, intelligi queant.“ Dass die Philologen ,veros esse Eruditos, id vero nego, atque pernego: Rimari Autorum Sensus, dignoscere scripta genuina a supposititiis, replere illorum lacunas, discernere variantes Lectiones, aliaque peragere, quae huc pertinent, labor est feré Herculeus, sed labor iste corticem tantum, ad nucleum usque non penetrat“. 
mit von der grammatischen Akribie vorbehaltloser Antikeverehrer. Mehr noch: Er machte die Übertreibungen der hartnäckigen Anciens sogar für das schwindende Ansehen der Gelehrtensprache verantwortlich (Merian 1743: 7). ${ }^{8}$ Andererseits widerlegte er die Gegner des Lateins mit topischen Argumenten aus Antike und Mittelalter, z. B. dem angeblich von Bernhard von Chartres erstmals verwendeten Riesen-Zwerg-Gleichnis, das Merian aus einer Rede des Genfer Theologen Jean-Alphonse Turrettini (1671-1737) übernahm (Merian 1743: 9; zur Motivgeschichte Merton 1980; Haug 1989), ${ }^{9}$ und mit der auf Plinius den Jüngeren zurückgeführten Behauptung, die Natur sei unerschöpflich, sie produziere stets mit gleicher Kraft (Kapitza 1981: 111, 223). ${ }^{10}$

Merian gelangte zur Annahme eines zwar fortwährenden, geschichtsphilosophisch aber unbestimmt bleibenden Fortschritts durch Kenntnisakkumulation und durch ständige Amelioration einmal erlangten Wissens. In der pro cathedra-Dissertation des Baslers halten sich die Argumente pro und contra Antike und humanistische Tradition in einem ambivalenten Konzept großer Zustimmung und entschiedener Kritik die Waage, das einerseits die Autorität der Alten relativiert und andererseits gerade auch unter Berufung auf antike Autoren neuerungs- und fortschrittsbefürwortende Auffassungen festigt. So steht Merian für das Latein als internationales Kommunikationsmedium der Gelehrten ein, möchte aber den Lateinunterricht von unnützem Ballast und grammatischem Kleinkram entlastet sehen (Merian 1743: $11 \mathrm{f}.) .{ }^{11}$ Mit Nachdruck gibt er im Sog der humanistischen Tradition den antiken Quellen (ad fontes!) im Vergleich mit den Übersetzungen, die die Qualität der Originaltexte nie erreichten, den Vorzug (Merian 1743: 6). ${ }^{12}$ Merian ist vom praktischen Nutzen

8 „Cum igitur maximi Zelotae, iique, qui de contemtu Linguae latinae quotidie queruntur, et clamitant, praecipuis ejusdem contemptus Autoribus meritò annumerentur [...].“

9 Jean-Alphonse Turrettini: De saeculo XVII. erudito et hodiernis literarum periculo oratio academica (14. Mai 1703). Genf 1704.

10 Plinius d. J., Epistolae 6, XXI: „Sum ex iis qui mirer antiquos, non tamen (ut quidam) temporum nostrorum ingenia despicio. Neque enim quasi lassa et effeta natura nihil iam laudabile pariat.“

11 „[...] rigidi illi Grammatici, quorum frons magisterialis et tetrica, joci et lusus omnis ignara, solo suo aspectu terrorem quaquaversus spargit, qui, si liceret regulas Etymologiae et Syntaxeos ferro et flammâ stabilirent, hoc vero prohibito ob levissimos quosque errores in subditorum terga atrocissimé saeviunt, eaque miserrimum in modum dolando stimulorum segetem efficiunt. [...] omnis culpa versatur in sinistris [...] docendi methodis, ut, si, ingenia nimia discendorum multitudine onerantur, si invertitur rerum ordo inchoando a difficilioribus, et progrediendo ad faciliora, docendo inutilia utilibus praetermissis [...].“

12 „Quod ad versiones, qui dissimilitudinem linguarum, praesertim antiquarum cum hodiernis probe habent perspectam, iis ignotum esse nequit, optimam quamque (sunt autem illae rarissimae) textum originalem nonnisi imperfecté exprimere.“ 
einer Universalsprache der Gelehrten überzeugt und will den Missständen im Lateinunterricht mit pädagogischen Maßnahmen, insbesondere mit der Auswahl geeigneter, ohne brachiale Erziehungsmittel wirkender Lehrkräfte, begegnen (Merian 1743: 12). Der deutschen Sprache fehle die elaborierte Terminologie; dies beweise die gelehrte Praxis, deutschen Texten lateinische Fußnoten als Erklärungshilfen beizugeben (Merian 1743: 3). ${ }^{13}$ In Basel dauerte es noch rund 85 Jahre, bis sich auch nur in einer medizinischen Qualifikationsschrift (Husner 1942: 240 spricht von einer „etwa der späteren Habilitationsschrift entsprechend[en]“ Publikation) das Deutsche erstmals durchsetzen konnte. Diese singuläre Tatsache bestätigt das allgemeine Bild der Sprachwahl im Hinblick auf die an Universitäten deutschsprachiger Länder eingereichten Promotionsschriften.

\section{Zwischenbilanz zur Theorie der Sprachwahl}

In den frühneuzeitlichen Thesenschriften protestantischer Hoher Schulen wurde, wie in Basel, die Sprachenfrage, häufiger seit dem letzten Viertel des 17. Jahrhunderts, zwar immer wieder kontrovers behandelt (Marti 1982: 675 [Register]), die Praxis des Lateingebrauchs erwies sich aber, wie einleitend vermerkt, als äußerst beharrlich, obwohl in den Reformdiskursen zu Beginn des 19. Jahrhunderts die Klagen insbesondere über mangelnde mündliche Sprachbeherrschung und ungenügende propädeutische Ausbildung der Disputanten markant zunahmen (Marti 1998: 61). Während die absolute Hegemonie des Lateins in den Dissertationen über die Frühe Neuzeit hinaus Bestand hatte, fehlen über den actus disputationis Informationen weitgehend (Füssel 2016), die allgemeine Schlüsse über den Sprachgebrauch, selbst für einen begrenzten regionalen Bereich, zulassen. Eher spärlich überlieferte Verlautbarungen stützen die Annahme, dass das mündliche Disputieren in lateinischer Sprache namentlich die Studenten, also Respondenten und Opponenten, auch schon im 17. Jahrhundert, bisweilen überforderte (Marti 1998: 54 f.; Füssel 2016: 44). Der Übergang vom Lateinischen zum Deutschen vollzog sich in den Dissertationen,

13 „Germanicè loquuti sunt, sed ita loquuti sunt, ut a nemine intelligerentur absque creberrimo indicis lat[ini] quem ad tractatuum suorum calcem semper adjecere, auxilio implorato“. Anderer Meinung war etwa zur selben Zeit der Philosophiehistoriker Jakob Brucker (16961770), der in einem Brief vom 1. Januar 1744 an den ,deutschen Literaturpapst‘ Johann Christoph Gottsched (Gottsched 2015: 464) wie Letzterer der Meinung war, „daß in unserer deutschen Sprache philosophieren eben so löblich und nüzlich seye, als Socrati in der Griechischen u. Ciceroni in der Lateinischen rühmlich gewesen.“ 
wie vorweggenommen, erst im Laufe des 19. Jahrhunderts und setzte sich in der Regel erst in dessen zweiter Hälfte durch. Dies sei im Folgenden an zwei Beispielen, in aller Kürze zunächst an der Friedrich-Wilhelms-Universität zu Berlin (heute Humboldt-Universität) und an einer Zusammenfassung zur ,Königlichen Universität $\mathrm{zu}$ Breslau' gezeigt. $\mathrm{Zu}$ beiden Institutionen liegen Verzeichnisse der Promovenden und der Dissertationen vor, die klare Schlussfolgerungen zur Entwicklung der Sprachwahl erlauben (Erman 1973; Pretzsch 1975).

\section{Der Wechsel vom Latein zum Deutschen in Dissertationen des 19. Jahrhunderts}

Der Bildungsmythos um Wilhelm von Humboldt und die Gründung der Berliner Universität, die im Oktober 1810 ihre Tore öffnete, aber erst am 29. April 1817 mit einer Feier inauguriert wurde, hat sich in den letzten Jahrzehnten universitätsgeschichtlicher Forschung verflüchtigt (Vom Bruch 2001). Die Bestimmungen zu den Promotionen in den Statuten von 1816 und das auch an der Berliner Universität wohl unerwartet späte Auftauchen deutschsprachiger Dissertationen bestätigen die damalige Traditionsgebundenheit des Graduierungswesens dieser Institution: Der Promovend musste, bevor er seine Dissertation ausarbeiten durfte, drei Studienjahre nachweisen und sich einem Examen unterziehen. Nach bestandener Prüfung hatte er die Pflicht, die in lateinischer Sprache und mit der schriftlichen Versicherung, er sei der Autor, eingereichte Dissertation drucken zu lassen. Letztere hatte er in einer öffentlichen lateinsprachigen Disputation zu verteidigen, und zwar in der theologischen, juristischen und philosophischen Fakultät ohne, in der medizinischen mit oder ohne Präses (Koch 1839: 60 f.). Ende 1817 wurde auch in der medizinischen Fakultät die letzte Präses-Respondentendisputation abgehalten (Erman 1973: 59 [Nr. 1109]). Neu an diesen Bestimmungen sind die grundsätzliche Anforderung, dass der Promovend als Doktorarbeit eine selbstständige wissenschaftliche Leistung vorzulegen habe, und das damit verbundene offizielle Verschwinden des Präses als Mit- oder Alleinverfassers der Inauguralschrift. In der theologischen Fakultät wurden bis 1883 nur lateinsprachige Dissertationen eingereicht (Erman 1973: 22-27) - ein Ausweichen auf das Deutsche war nicht vorgesehen. Den Medizinern gestattete ein Ministerialerlass vom 22. Mai 1867 auch deutschsprachige Doktorarbeiten, für die philosophische Fakultät wurde gleichzeitig für Promotionsschriften in den Fachbereichen klassische und orientalische Philologie, Altertumskunde, Geschichte und antike Philosophie 
weiterhin Latein als obligatorisch erklärt, am 5. November 1879 der Fakultät dann aber die Befugnis übertragen, Deutsch auch in Dissertationen zur mittelalterlichen und neuzeitlichen Geschichte zuzulassen. Wer sich für Deutsch entschied, hatte aber in einer mündlichen Promotionsprüfung ausreichende Lateinkenntnis durch Interpretation eines Texts eines römischen $\mathrm{Au}$ tors nachzuweisen. Die Zulassung zur mündlichen Disputation war also vom Resultat dieses Examens abhängig. Am 14. Juli 1876 erlaubte ein Ministerialerlass die freie Wahl zwischen Deutsch und Latein für juristische Promotionsschriften und setzte die ausschließliche Verwendung des Deutschen in der mündlichen Verteidigung der Abhandlung, in der juristischen Disputation, fest. ${ }^{14}$

Welches waren die unmittelbaren Auswirkungen der damals zugestandenen Freiheit der Sprachwahl in den drei unteren Fakultäten der Berliner Universität? In der medizinischen Fakultät wurden 186771 Dissertationen in lateinischer und 62 in deutscher Sprache eingereicht, im Jahr darauf waren es bereits 110 deutschsprachige und nur noch sechs lateinsprachige, 1869 und 1870 waren alle medizinischen Promotionsschriften in Deutsch verfasst (Erman 1973: 477-517). Der abrupte Wechsel von Latein zu Deutsch hinterlässt den Eindruck, die Promovenden seien durch den Regierungserlass von einer schwer erträglichen Belastung befreit worden. Nicht von ungefähr betrafen die Klagen über mangelnde Lateinkenntnis in erster Linie die Mediziner (Marti 1998: $54 \mathrm{f}$.). Von 1877 bis 1885 entstanden in Berlin lediglich 42 juristische Dissertationen, 35 in deutscher und nur sieben in lateinischer Sprache; in den letzten vier Jahren dieser Promotionsperiode (1882-1885) war noch eine einzige, allerdings im Jahr 1885 verteidigte Dissertation in Latein verfasst (Erman 1973: 48-51). Die auf die Mediziner zutreffende Beobachtung, mit der Preisgabe des Lateinobligatoriums einem Bedürfnis der Zeit entsprochen zu haben, bestätigt sich in etwas geringerem Maße für die Juristen. Wie der referierte Regierungsbeschluss von 1867 nahelegt, ist aber bei der philosophischen Fakultät hinsichtlich der Sprachwahl von differenzierteren Befunden auszugehen. In der Übergangsperiode von 1867 bis 1883 waren die naturwissenschaftlichen Fächer, allen voran die Chemie, die aufstrebenden Promotionsdisziplinen, die auch dem Aufschwung des Deutschen als Dissertationssprache den kräftigsten Schub verliehen (Erman 1973: 679-714). Von den 35 Doktorarbeiten im Jahr 1880 waren 27 in Deutsch abgefasst, davon neun in Chemie, fünf in Mathematik, je zwei in Physik, Wirtschaftswissenschaft und Botanik, je eine in Biologie,

14 Daude (1887: 77, 111, 148, 187) (Paragraphen in den einschlägigen Fakultätsstatuten von 1838 mit den in unserem Zusammenhang wesentlichen ergänzenden Fußnoten zu den im Laufe der Zeit veränderten Bestimmungen). 
Astronomie, Paläontologie, Philosophie, älterer französischer Literatur, älterer englischer Literatur und in Geschichte. Lateinsprachige Dissertationen schrieben im selben Jahr einzig die acht Altphilologen (Erman 1973: 698-701), 1879 waren es noch fünf ( $25 \%$ ) von insgesamt 20, die Arbeit eines Mathematikers, eine zur deutschen Sprachwissenschaft, eine zur Literatur des englischen Mittelalters und die beiden Promotionsschriften in Altphilologie. Deutsch abgefasst waren je drei Dissertationen in Chemie, Mathematik und in den neueren Philologien, je zwei in Physik und Botanik, je eine in Philosophie und in Geschichte (Erman 1973: 696-698). Die historiographisch allenthalben in den Vordergrund gerückte Medizinerschwemme, die im Laufe des 18. Jahrhunderts begann und im 19. Jahrhundert einen Höhepunkt erreichte (Müller 2003), betraf vor allem die Spätgründung Berlin, wo denn auch inneruniversitär die fakultätsbezogene Priorisierung des Deutschen ihren Anfang nahm.

Das Promotionswesen entwickelte sich im 19. Jahrhundert an der Universität des im Jahr 1741 preußisch gewordenen Breslau nicht unabhängig von dem der Universität Berlin, obwohl die Breslauer Universitätsgeschichte ganz anders als die der Berliner Hochschule verlaufen war. Dem Stiftungsbrief des böhmisch-ungarischen Königs Wladislaw II. von 1505 war kein Erfolg beschieden, da Papst Julius II. einer Universitätsgründung nicht zustimmte, sondern der Standort Frankfurt/Oder bevorzugt wurde. 1639 errichteten die Jesuiten in Breslau ein Kollegium, dem am 21. Oktober 1702 in Wien von Kaiser Leopold I. das Universitätsprivileg für eine theologische und eine philosophische Fakultät verliehen wurde. In der preußischen Zeit bestand die Leopoldina als katholische Landesuniversität unter jesuitischer Leitung weiter. Am 24. April 1811 wurde die Frankfurter Viadrina mit der Breslauer Leopoldina vereinigt, am folgenden 19. Oktober die Königliche Universität zu Breslau gegründet, die 1911 in Friedrich-Wilhelms-Universität umbenannt wurde (Conrads 1983: 91-94; Herzig 2004).

In einer Einladungsschrift von 1845 zur Feier des Geburtstags des preußischen Königs Friedrich Wilhelm IV. plädierte der Breslauer Altphilologe Karl Ernst Christoph Schneider (1786-1856) für eine freie Sprachwahl der Universitätsdozenten und setzte die Beherrschung des Lateins als Beurteilungskriterium wissenschaftlicher Leistung grundsätzlich außer Kraft. Auch für Inauguraldissertationen verlangte er, ohne ausdrücklich nach Fachbereichen zu differenzieren, die Aufhebung des Lateinobligatoriums. Fehlende lateinische Sprachkompetenz wirke sich auf die Qualität wissenschaftlicher Publikationen negativ aus. Selbst den Erfolg im Griechischstudium machte Schneider nicht von Lateinkenntnissen abhängig. Er stellte einen Fortschritt des Deutschen als Sprache der Gebildeten fest, den er an den Werken Johann Gottfried Herders, Gotthold Ephraim Lessings und Johann Joachim Winckelmanns illustrierte. Die 
Notwendigkeit, in der Wissenschaft von der lateinischen zur deutschen Sprache zu wechseln, begründet Schneider mit der Unübersetzbarkeit der kantschen Transzendentalphilosophie: Sie könne nicht in eine der Norm römischer Latinität genügende sprachliche Form gegossen werden. Die erwähnten Exempel zeichnen eine irreversible Entwicklung nach und relativieren, wenigstens in theoria, die Berechtigung des universitären Lateinmonopols.

Die Initiative des Breslauer Altphilologen, die vor allem mit den zeitgenössischen Anforderungen des philosophischen Denkens, insbesondere mit den Anfängen des deutschen Idealismus, zusammenhängt, bewegt sich freilich dennoch, obwohl in moderaterer Form und aufgrund pragmatischer Überlegungen, im geistigen Horizont der von ihm mit Überzeugung vertretenen humanistischen Tradition. ${ }^{15}$ Mit seinem sprachlichen Plädoyer versuchte er, ohne es auszusprechen, ihre Nachwirkung innerhalb des eigenen Fachs zu sichern und den übrigen Universitätsbetrieb von einer Anforderung zu befreien, welche die Qualität wissenschaftlicher Leistung in den nichtaltphilologischen Fächern weiter schwächen würde.

Wilhelm Erman (1850-1932), der Verfasser des Berliner Dissertationenverzeichnisses, zunächst Leiter der Berliner Universitätsbibliothek, wurde 1901 als Direktor der Universitätsbibliothek nach Breslau berufen (Erman 1994: 218234). Bald setzte er sich für die Anfertigung einer Bibliographie Breslauer Dissertationen nach dem Berliner Muster ein, mit der er seinen Mitarbeiter Karl Pretzsch (1863-1943) beauftragte, der, noch bevor Erman seine Stelle in der schlesischen Kapitale antrat, bereits von Berlin an die Universitätsbibliothek Breslau versetzt worden war (Erman 1994: 184, 235 f.). Diese personellen Verflechtungen erleichtern die Auswertung des Breslauer Dissertationenverzeichnisses und den Vergleich der Einträge beider Bibliographien. Die statutarischen Breslauer Bestimmungen, die den Berliner Vorschriften über die Erlangung akademischer Würden sehr ähnlich sind, brauchen nicht wiederholt zu werden (Koch 1839: 318-341; Statuten vom 21. Februar 1816). In Breslau waren sowohl an der protestantischen wie an der katholischen theologischen Fakultät und an der philosophischen Fakultät der Lizentiaten- und der Doktortitel zu erwerben, an der juristischen und der medizinischen nur der Doktorgrad. 1885 wurde - ein Ausnahmefall - in der evangelischen Theologie ein Lizentiat in

15 Kaufmann (1911: 236) erwähnt Schneiders Vorschlag und einen Vorstoß des Philologen und Archäologen Joseph Julius Athanasius Ambrosch (1804-1856), der sich auf Schneiders Programmschrift berief und den Professoren bei amtlichen Reden und Schriften die Wahl der deutschen oder der lateinischen Sprache überlassen wollte, was der Senat aber ablehnte. Ausgerechnet die Mediziner Wilhelm Hermann Georg Remer (1775-1850) und Traugott Wilhelm Gustav Benedict (1785-1862) lehnten damals die freie Sprachwahl ab. 
deutscher Sprache vergeben (Pretzsch 1975: 28), die übrigen theologischen Promotionsschriften waren bis zu diesem Jahr alle in lateinischer Sprache abgefasst. In der juristischen Fakultät erfolgte 1876 aufgrund des erwähnten Regierungserlasses ein abrupter Wechsel vom Lateinischen zum Deutschen (Pretzsch 1975: 44), in der medizinischen wurden, wie in Berlin, infolge der genannten Verordnung ab dem 20. Juli 1867 nur noch deutschsprachige Dissertationen (Pretzsch 1975: 127-169), vor diesem Datum im selben Jahr noch vier in Latein eingereicht (Pretzsch 1975: 126). Wie in Berlin sind auch in Breslau die Verhältnisse an der philosophischen Fakultät am kompliziertesten: Von 1850 bis 1856 gab es bereits Dissertationen in deutscher Sprache (1853 vier von neun, 1854 drei von sechs, 1856 eine von sieben), von 1858 bis 1866 lediglich lateinsprachige. Von da an vermehrte sich der Anteil der deutschsprachigen im Laufe der Zeit, aber nicht linear. 1873 war die Wahl der Sprache fast ausgeglichen (10 Deutsch, 9 Latein), 1874 und 1875 überwog das Latein (1874: 22:15; 1875: 18:16), ab 1876 das Deutsche, mit Ausnahme von 1880 (11 Latein, 10 Deutsch). 1885 kamen auf insgesamt 20 Dissertationen immer noch acht in lateinischer Sprache. Immerhin wurde 1873, 1882 und 1884 an der philosophischen Fakultät je eine in Englisch verfasste Dissertation abgeliefert. Die auf die Berliner Universität zutreffenden Feststellungen zur Sprachwahl lassen sich infolge der damaligen Zugehörigkeit Schlesiens zu Preußen zwar im Allgemeinen auf die Universität Breslau übertragen, im Einzelnen sind trotz derselben Gesetzesvorgaben Unterschiede in der praktischen Auswirkung der Bestimmungen auszumachen. Von den $29 \mathrm{im}$ Jahr 1879 eingereichten philosophischen Dissertationen sind 22 in deutscher und sieben, ausschließlich altphilologische, in lateinischer Sprache verfasst, unter den deutschsprachigen befinden sich 18 naturwissenschaftliche (einschließlich vier über mathematische Themen) mit der, wie in Berlin, festgestellten Dominanz der Chemie (neun). Im Jahr darauf sind es zehn deutsch- und elf lateinsprachige; letztere entfallen alle auf die Altphilologie. 1881 änderte sich das Verhältnis massiv zugunsten des Deutschen (14:3), unter den deutsch verfassten Dissertationen befinden sich sieben nicht naturwissenschaftliche (Philosophie, Mediävistik, neuere Philologien). Ein ähnliches Bild vermitteln die Zahlen des folgenden Jahres (19 Deutsch, 10 Latein), in dem ebenfalls ein Anstieg deutschsprachiger Dissertationen in philosophisch-historischen Fächern (vor allem in Philosophie und Geschichte) zu verzeichnen und Latein wiederum ganz auf die Promotionen der Altphilologen beschränkt ist.

In Berlin und in Breslau ist die Ablösung des Lateins durch das Deutsche als Dissertationssprache in der philosophischen Fakultät ein erst im späten 19. Jahrhundert einsetzender, vor allem von den Naturwissenschaften, insbesondere dem Fach Chemie, ausgehender Vorgang, der dann aber, durch obrig- 
keitliche Erlasse befördert, in allen drei unteren Fakultäten zum raschen Wandel der Sprachwahl führte. Die für die Übergangszeit selektiv ermittelten, auf Promotionsfächer und -themen bezogenen Befunde legen nahe, die Ergebnisse durch weitere, detaillierte Nachforschungen $\mathrm{zu}$ überprüfen und im Hinblick auf die gegenüber dem Gebrauch der Muttersprache resistenten Fachbereiche (Theologie und klassische Philologie) auf die folgenden Jahrzehnte auszuweiten. Für das Promotionswesen des 19. Jahrhunderts bleibt das eingangs hervorgehobene Beharrungsvermögen des Lateins als Dissertationssprache noch einmal festzuhalten. Die politisch begründete Kompatibilität der beiden Dissertationenverzeichnisse der preußischen Universitäten Berlin und Breslau schließt allerdings das Risiko ein, der Pluralität des Promotionswesens im gesamten deutschen Sprachgebiet nicht angemessen Rechnung zu tragen. Die Veränderung der Sprachwahl muss daher für weitere (deutsche) Hochschulen untersucht werden. An der Feststellung, dass die Zäsur in der zweiten Hälfte des 19. Jahrhunderts zu lokalisieren ist, dürfte sich kaum Wesentliches ändern.

\section{Fazit und Ausblick}

Es wurde hier absichtlich der Begriff ,Wissenschaftssprache“ sehr zurückhaltend verwendet, da er die schwierige Bestimmung dessen voraussetzt, was unter ,Wissenschaft' $\mathrm{zu}$ verstehen ist. Bevorzugt wurden daher die für die Frühe Neuzeit adäquateren Bezeichnungen ,Gelehrter‘, ,Gelehrsamkeit‘ und ,Gelehrtenliteratur', zu deren Kernbestand die Disputationsschriften zählen. Heute ist weitgehend unbestritten, dass auch in den Naturwissenschaften von der Historizität der Erkenntnisse auszugehen (Oexle 2004 geht nicht von einer Selbstverständlichkeit aus, anders Breidbach 2011), das Denken in Modellen und Vorläufigkeiten stark zu machen und die Vorstellung preiszugeben sei, Wissenschaft generiere unveränderliche Wahrheiten. ${ }^{16}$ Trotzdem spielen teleologische Erklärungsmuster und Verlaufsmodelle, die von einem Erkenntnisfortschritt ausgehen, sowohl in Bezug auf Entstehung und Gebrauch von Wissenschaftssprachen als auch in der Geschichtsschreibung zur ars disputandi nach wie vor eine zentrale Rolle. Aus disputationsgeschichtlicher Warte seien nun thesenartig einige Aspekte der fable convenue zur Diskussion gestellt (vgl. Marti 2016b).

Vorwegzunehmen ist, dass sich die frühneuzeitlichen Disputanten meist sowohl vom philosophischen Skeptizismus als auch von dem als logikfeind-

16 Büchel (1992: 224): „Die Vorläufigkeit und Widerruflichkeit, die somit dem naturwissenschaftlichen Beitrag zur menschlichen Weltorientierung anhaften, sind aber wohl nicht größer als die Vorläufigkeit und Widerruflichkeit allen menschlichen Erkenntnisbemühens.“ 
lich, ja als häretisch eingestuften Spiritualismus der Mystik abgrenzten, insoweit er die natürliche Vernunft als Erkenntnisorgan außer Kraft setzte und allein göttlicher Inspiration das Wort redete. Logik und Rhetorik waren die Instrumente gelehrter Begriffsbildung und Argumentation, das Latein blieb, wie gezeigt, in den frühneuzeitlichen Dissertationen ususgemäß das Medium gelehrter Kommunikation.

Die frühneuzeitliche disputatio beschäftigte sich per definitionem mit Wahrscheinlichkeitserkenntnissen und erklärte sich infolgedessen für Fragen und Themen zuständig, die ein Pro und ein Kontra, einen status controversiae, zuließen. Die wichtigsten wissenstheoretischen Grundlagen lieferte für das Disputieren lange Zeit die Philosophie des Aristoteles und von dessen Anhängern, insbesondere die Topik, die in der Metaphysik überlieferten genera causarum sowie die Lehre der Syllogismen in der Analytica priora. Das probabilistische Wissenskonzept führte gelegentlich zu Legitimationskrisen der disputatio, die in der Frühen Neuzeit aber mit der Integration mathematischer, empiristischer und anderer Erkenntnismethoden mehr oder weniger erfolgreich aufgefangen wurden. Über die im weiten Sinn verstandenen technischen Künste wurde recht selten disputiert; dennoch ließen bereits im frühen 17. Jahrhundert Präsides vereinzelt Dissertationen über die artes mechanicae, die empirische Erforschung der Natur und über naturwissenschaftliche Mess- und Beobachtungsinstrumente verteidigen. Die Ansicht einer allgemeinen Wendung in der Philosophie von der Theorie und von deren angeblicher Kerndisziplin, der Metaphysik, zu den praktischen Fächern, namentlich zur Politik, die vor allem Ende des 17. Jahrhunderts stattgefunden haben soll, ist nicht zutreffend.

Die undifferenzierte Auffassung der frühneuzeitlichen Universität als bloßer Lehranstalt, die seit dem Mittelalter unkritisch herkömmliches Wissen tradiere und erst im 19. Jahrhundert kreativ Forschungsresultate hervorgebracht und vermittelt habe, lässt sich in dieser Allgemeinheit ebenso wenig aufrechterhalten und ist denn inzwischen auch widerlegt. Reinhart Kosellecks Sattelzeithypothese und William Clarks (2006) universitätsgeschichtliches Standardwerk, die von einer solchen Entwicklung ausgehen, sind von der Dissertationenforschung zu heuristischen Zwecken zwar heranzuziehen, verlieren aber im Licht der Geschichte der ars disputandi, auf die sich Clark wesentlich stützt, an historischer Überzeugungskraft (Joas \& Vogt 2011, Fulda 2016, Marti 2016b). Dies beweisen die im 19. Jahrhundert fortgesetzte, bisweilen einseitig dem Weiterleben des Mittelalters zugeschriebene Verwendung der lateinischen Sprache wie auch frühneuzeitliche Dissertationen, insbesondere der Universität Halle, die vor der angesprochenen Zeitenwende Novitätsund Fortschrittsansprüche erfüllten (Marti 2014a). Vor allem medizinische Thesenschriften waren oft Transportmittel empirischer Beobachtungen und 
anderer, in ihrer Zeit aktueller Forschungsergebnisse. Daher ist auch dem strikten Gegensatz von traditionsgebundenen Universitäten auf der einen und dem wissenschaftlichen Fortschritt zugewandten Akademien auf der anderen Seite der historische Boden entzogen. Dasselbe gilt für die oft plakativ formulierte Mutation des auf bloßes Sammeln festgelegten frühneuzeitlichen Gelehrten zum entdeckenden und forschenden Wissenschaftler der späteren Zeit (Steiner 2009: 125-136: Kommunikationsgeschichte als Geschichte eines sozialen Typus: Vom „Gelehrten“ zum „Wissenschaftler“). Schließlich besitzt die krude Opposition von deutschsprachiger, fortschrittsfreudiger Aufklärung und durchweg auf Rückschritt abonnierter lateinsprachiger frühneuzeitlicher Gelehrsamkeit nicht den oft vorausgesetzten (geschichtlichen) Rückhalt. Nicht nur Hallenser und Göttinger Dissertationen widerlegen diese einfachen wissensgeschichtlichen Konnotationen, auch Thesenschriften vor allem aus Jena, Leipzig, Wittenberg, Altdorf, Königsberg und Straßburg. Die Ausweitung der Adressaten- und Rezipientenkreise dank der Produktion vernakulärer Druckschriften bleibt für das 18. Jahrhundert unbestritten, den wechselseitigen - und das ist $\mathrm{zu}$ betonen - Wissenstransfer von der einen in die andere Sprache gilt es für das Zeitalter der Aufklärung noch zu erforschen.

Nicht erst seit von den Promovenden in Dissertationen ausdrücklich eigenständige Forschungsleistungen verlangt wurden, zählten die Thesenschriften zu den Plattformen des im Sinn ihrer Zeit verstandenen wissensgeschichtlichen Fortschritts. Namentlich die von den Professoren verfassten und von Studenten bloß verteidigten Dissertationen vermittelten nicht selten neueste Erkenntnisse:

Dann viele Gelährten kommen bey Untersuchung der Warheit auf neue Gedancken, wollen aber deswegen nicht gleich gantze Bücher und Tractate schreiben, sondern geben ihre neue Meinung in forma Disputationis heraus. (Apin 1719: 24)

Dies geschah auch an Hohen Schulen, die kein Promotionsprivileg besaßen. Es sei nur an die Mitte des 18. Jahrhunderts verteidigten paläontologischen Dissertationen des Zürcher Naturforschers Johannes Gessner (1709-1790) erinnert, der an dem bis vor kurzem im Ruf einer Heimstätte verknöcherter reformierter Orthodoxie stehenden Carolinum Naturkunde unterrichtete (Leu 2016).

Wie Ewald Horn stellte der Breslauer Geschichtsprofessor und Universitätshistoriker Georg Kaufmann (1842-1929) den allermeisten zeitgenössischen Dissertationen ein katastrophales Zeugnis aus und wollte den Publikationszwang für Promotionsschriften abschaffen:

Denn die wissenschaftlichen Forderungen für die Promotion blieben doch immer Schülerleistungen, wenn auch verbunden mit dem Specimen doctrinae der Dissertation, in der sich der bisherige Schüler auf einem engeren Gebiete als selbständiger Forscher erweisen 
soll. Da diese Selbständigkeit jedoch nicht selten an den Krücken des Professors gegangen ist, oder da die Untersuchung nicht selten zu einer Reihe von anderen gehört, die als Muster dienten, so liegt in den mit allem gelehrten Apparat versehenen Dissertationen, auch wenn sie unsere Kenntnis erweitern, nicht selten nur ein geringer Beweis für den Grad wissenschaftlicher Selbständigkeit, der mit dem anspruchsvollen Titel ausgedrückt zu werden scheint. [...] Da der Durchschnitt unserer Studierenden zu wirklich selbständiger wissenschaftlicher Arbeit nicht geeignet ist, so kann die Promotion niemals zur mehr oder weniger allgemeinen Regel werden, ohne zu sinken. [...] Es füllen sich seitdem die Säle der Bibliotheken jährlich mit so und so viel Kubikmetern von Dissertationen, die nur selten aus ihrem Versteck herausgeholt werden. [...] Bei einem großen Teile dieser Arbeiten würde es genügen, das Ergebnis in einer Zeitschrift oder einem Sammelwerk zu verzeichnen. (Kaufmann 1911: 249)

Die Promotion sollte, so Kaufmann (1911: 250), nur den zu einer akademischen Karriere Qualifizierten offenstehen. Aus universitäts- und wissensgeschichtlicher Sicht deckt sich freilich der Quellenwert einer Dissertation nicht mit deren inhaltlicher Qualität, da die Promotionsschriften auf breiter Basis Aufschluss über den Unterricht, die Generierung und Rezeption von Wissensbeständen und über wissenschaftliche Ausrichtungen im Ganzen geben. Ob die beklagte, angeblich schlechte Qualität der Dissertationen eine Folge der geforderten selbstständigen Arbeitsweise der Promovenden und des Endes der Präsesdissertation ist, muss hier offen bleiben.

Die Erforschung frühneuzeitlicher Dissertationen verabschiedet beweiskräftig eindimensional angelegte Modernisierungstheorien, teleologische Erklärungsmodelle, historiographische Großerzählungen überhaupt, und fordert zumindest Präzisierungen allgemein wissens- respektive wissenschaftsgeschichtlicher Behauptungen und Selbstverständlichkeiten ein. Dadurch gewinnt die permanente Gleichzeitigkeit des Unterschiedlichen respektive die Gleichzeitigkeit des Ungleichzeitigen als historiographische Leitvorstellung an Geltung (Landwehr 2012; Marti 2016b: 297). Der zeitweise Abschied von der auf ,Höhenkämme‘ oder Geistesheroen ausgerichteten Wissenschaftsgeschichte ist nicht bedauerlich, im Gegenteil, aus rezeptionsgeschichtlichem Blickwinkel und hinsichtlich der gewünschten Differenzierungen ein Vorzug.

Im Epilog einer Einführung zur Wissenschaftstheorie wird hervorgehoben, dass die besseren Argumente allemal die Grundlage einer verlässlichen Problemlösungsstrategie bilden (Poser 2001: 294). Das beste Argument kannten oder suchten, auf doch recht verschiedenen Wegen, auch die an frühneuzeitlichen Disputationen beteiligten Protagonisten. Die vermehrten Erfahrungen mit frühneuzeitlichen Dissertationen legen nahe, dass von einem weiten Wissensrespektive Wissenschaftsbegriff auszugehen sei, der für viele Formen von Rationaliät offen ist, daher weiterhin das Risiko fehlender semantischer Exaktheit eingeht und den Dialog über das Wesen, die Mittel und die Ziele der Wissen- 
schaften fortsetzt. Die Antwort auf die Frage, was unter ,Wissenschaftssprache“ $\mathrm{zu}$ verstehen ist, muss die Diskussion methodischer Prämissen und die historische Kritik von Darstellungsnormen einbeziehen. Wie dies aus sprachwissenschaftlicher Perspektive geschehen könnte, steht auf einem anderen Blatt. Jedenfalls wird der Sprachhistoriker die frühneuzeitlichen Dissertationen als Arsenale der Begriffsprägung und -vermittlung, zur Sammlung von Belegen, selbst für die Geschichte der Landessprache, mit Gewinn heranziehen. Das Archiv der Begriffsgeschichte wird von den bislang selten konsultierten Thesenschriften profitieren. Hier begnüge ich mich mit dem Hinweis auf ein Einzelbeispiel, eine Hallenser Dissertation (Lerche 1730/1983), die den Übersetzern des Lateins in die deutsche Sprache Schwierigkeiten bereitete, aber für die Geschichte paläontologischer und geologischer Begriffe, nicht zuletzt wegen den fachterminologischen Einsprengseln in deutscher Sprache, einen beachtlichen Dokumentationswert besitzt. Hiermit sind wir bei der sogenannten empirischen Sprachforschung angelangt. Ob sie gewillt ist, die frühneuzeitlichen lateinsprachigen Dissertationen vermehrt in ihr Quellenrepertoire aufzunehmen (einen Anfang machte verdienstvoll im Feld der Meteorologie Klein 1999), wird sich zeigen. Ich empfehle, es mit Bedacht wiederholt $\mathrm{zu}$ tun.

\section{Bibliographie}

Alvermann, Dirk (2007): Greifswalder Universitätsreformen 1648-1815. In: Dirk Alvermann, Nils Jörn \& Jens E. Olesen (Hrsg.), Die Universität Greifswald in der Bildungslandschaft des Ostseeraums. Berlin: Lit., 69-104.

Apin, Sigmund Jakob (1719): Unvorgreiffliche Gedancken / wie man so wohl Alte als Neue DISSERTATIONES ACADEMICAS mit Nutzen sammlen / und einen guten INDICEM darüber halten soll. Nürnberg, Altdorf: Johann Daniel Tauber.

Beetz, Manfred (1980): Rhetorische Logik. Prämissen der deutschen Lyrik im Übergang vom 17. zum 18. Jahrhundert. Tübingen: Max Niemeyer.

Beims, Klaus-Dieter (2015): Antike Texte an christlichen Schulen. Die römischen Autoren im Lateinunterricht des Halleschen Pietismus. Halle: Franckesche Stiftungen.

Borst, Arno (1995): Der Turmbau von Babel. Geschichte der Meinungen über Ursprung und Vielfalt der Sprachen und Völker. 6 Bde. München: dtv.

Breidbach, Olaf (2011): Radikale Historisierung. Kulturelle Selbstversicherung im Postdarwinismus. Berlin: Suhrkamp.

Büchel, Wolfgang (1992): Naturwissenschaften. In: Helmut Seiffert \& Gerard Radnitzky (Hrsg.), Handlexikon der Wissenschaftstheorie. München: dtv, 222-231.

Clark, William (2006): Academic Charisma and the Origins of the Research University. Chicago, London: The University of Chicago Press.

Conrads, Norbert (1983): Breslau (Wroctaw). In: Laetitia Boehm \& Rainer A. Müller (Hrsg.), Universitäten und Hochschulen in Deutschland, Österreich und der Schweiz. Eine Universitätsgeschichte in Einzeldarstellungen. Düsseldorf: Econ, 91-94. 
Daude, Paul (1887): Die Königl. Friedrich=Wilhelms=Universität zu Berlin. Systematische Zusammenstellung für dieselbe bestehenden gesetzlichen, statutarischen und reglementarischen Bestimmungen. Berlin: H. W. Müller.

Döring, Detlef (2007): Gelehrte Gesellschaften in Pommern im Zeitalter der Aufklärung. In: Dirk Alvermann, Nils Jörn \& Jens E. Olesen (Hrsg.), Die Universität Greifswald in der Bildungslandschaft des Ostseeraums. Berlin: Lit., 123-153.

Eco, Umberto (1994): Die Suche nach der vollkommenen Sprache. Aus dem Italienischen von Burkhart Kroeber. München: Beck.

Erman, Wilhelm (1973): Verzeichnis der Berliner Universitätsschriften 1810-1885. Nebst einem Anhang enthaltend die ausserordentlichen und Ehren-Promotionen. Hildesheim, New York: Georg Olms.

Erman, Wilhelm (1994): Erinnerungen. Bearbeitet und hrsg. von Hartwig Lohse. Köln u. a.: Böhlau.

Fleck, Ludwik (1980): Entstehung und Entwicklung einer wissenschaftlichen Tatsache: Einführung in die Lehre vom Denkstil und Denkkollektiv, 1. Aufl. Frankfurt am Main: Suhrkamp.

Fulda, Daniel (2016): Sattelzeit. Karriere und Problematik eines kulturwissenschaftlichen Zentralbegriffs. In: Elisabeth Décultot \& Daniel Fulda (Hrsg.), Sattelzeit. Historiographische Revisionen. Berlin, Boston: De Gruyter, 1-16.

Füssel, Marian (2016): Die Praxis der Disputation. Heuristische Zugänge und theoretische Deutungsangebote. In: Marion Gindhart, Hanspeter Marti \& Robert Seidel (Hrsg.), Frühneuzeitliche Disputationen. Polyvalente Produktionsapparate gelehrten Wissens. Köln u. a.: Böhlau, 27-48.

Gesner, Johann Mattias (2013): Institutiones rei scholasticae - Leitfaden für das Unterrichtswesen. Hrsg. und übersetzt von Meinolf Vielberg. Wiesbaden: Harrassowitz.

Gottsched, Johann Christoph (2015): Briefwechsel unter Einschluß des Briefwechsels von Luise Adelgunde Victorie Gottsched. Bd. 9: November 1742-Februar 1744. Hrsg. und bearbeitet von Detlef Döring, Franziska Menzel, Rüdiger Otto \& Michael Schlott. Berlin, Boston: De Gruyter.

Grimm, Gunter E. (1983): Von der ,politischen“ Oratorie zur ,philosophischen“ Redekunst. Wandlungen der deutschen Rhetorik in der Frühaufklärung. In: Rhetorik. Ein internationales Jahrbuch 3, 65-96.

Haug, Walter (1989): Die Zwerge auf den Schultern von Riesen. Epochales und typologisches Geschichtsdenken und das Problem der Interferenzen. In: Walter Haug, Strukturen als Schlüssel zur Welt: kleine Schriften zur Erzählliteratur des Mittelalters. Tübingen: Max Niemeyer, 86-109.

Herzig, Arno (2004): Die Vereinigung von Leopoldina und Viadrina 1811. In: Norbert Conrads (Hrsg.), Die tolerierte Universität. 300 Jahre Universität Breslau 1702 bis 2002. Katalogbuch zur Ausstellung „Die tolerierte Universität“. Stuttgart: Franz Steiner, 244-254.

Horn, Ewald (1893): Die Disputationen und Promotionen an den Deutschen Universitäten vornehmlich seit dem 16. Jahrhundert. Mit einem Anhang enthaltend ein Verzeichnis aller ehemaligen und gegenwärtigen deutschen Universitäten. Leipzig: Harrassowitz.

Husner, Fritz (1942): Verzeichnis der Basler medizinischen Universitätsschriften von 15751829. In: Festschrift für Jacques Brodbeck-Sandreuter [...] zu seinem 60. Geburtstag. Basel [ohne Verlagsangabe], 137-269.

Joas, Hans \& Peter Vogt (Hrsg.) (2011): Begriffene Geschichte. Beiträge zum Werk Reinhart Kosellecks. Berlin: Suhrkamp. 
Kapitza, Peter K. (1981): Ein bürgerlicher Krieg in der gelehrten Welt. Zur Geschichte der Querelle des Anciens et des Modernes in Deutschland. München: Wilhelm Fink.

Kaufmann, Georg (Hrsg.) (1911): Festschrift zur Feier des hundertjährigen Bestehens der Universität Breslau. Erster Teil. Geschichte der Universität Breslau 1811-1911. Breslau: Ferdinand Hirt.

Klein, Wolf Peter (1999): Die Geschichte der meteorologischen Kommunikation in Deutschland. Eine historische Fallstudie zur Entwicklung von Wissenschaftssprachen. Hildesheim u. a.: Olms.

Koch, Johann Friedrich Wilhelm (1839): Die Preußischen Universitäten. Eine Sammlung der Verordnungen, welche die Verfassung und Verwaltung dieser Anstalten betreffen. Erster Band. Die Verfassung der Universitäten im Allgemeinen. Berlin u. a.: Ernst Siegfried Mittler.

Landwehr, Achim (2012): Von der ,Gleichzeitigkeit des Ungleichzeitigen‘. In: Historische Zeitschrift 295, 1-34.

Lerche, Johann Jakob (1730/1983): Oryctographia Halensis (1730). Halles älteste Regionalgeologie ins Deutsche übersetzt von Horst Koehn. Mit Einleitung und Kommentar hrsg. v. Heinz Pfeiffer \& Max Schwab. Halle: Martin-Luther-Universität Halle-Wittenberg.

Leu, Urs B. (2016): „Praeterit enim Species hujus Mundi“. Die paläontologischen Zürcher Dissertationen von Johannes Gessner (1709-1790). In: Marion Gindhart, Hanspeter Marti \& Robert Seidel (Hrsg.), Frühneuzeitliche Disputationen. Polyvalente Produktionsapparate gelehrten Wissens. Köln u. a.: Böhlau, 229-253.

Ludovici, Jakob Friedrich (Präses) \& Christian Leyssner (Respondent) (1720): Dissertatio juridica de grammatica mala. Oktober 1720. Halle: Christian Henckel.

Marti, Hanspeter (1982): Philosophische Dissertationen deutscher Universitäten 1660-1750. Eine Auswahlbibliographie. Unter Mitarbeit von Karin Marti. München u. a.: K. G. Saur.

Marti, Hanspeter (1994a): Disputation. In: Gert Ueding (Hrsg.), Historisches Wörterbuch der Rhetorik, Bd. 2. Tübingen: Max Niemeyer, 866-879.

Marti, Hanspeter (1994b): Dissertation. In: Gert Ueding (Hrsg.), Historisches Wörterbuch der Rhetorik, Bd. 2. Tübingen: Max Niemeyer, 880-884.

Marti, Hanspeter (1998): Lateinsprachigkeit - ein Gattungsmerkmal der Dissertationen und seine historische Konsistenz. In: Jahrbuch für Internationale Germanistik 30 (1), 50-63.

Marti, Hanspeter (2001): Dissertation und Promotion an frühneuzeitlichen Universitäten des deutschen Sprachraums. Versuch eines skizzenhaften Überblicks. In: Rainer A. Müller (Hrsg.), Promotionen und Promotionswesen an deutschen Hochschulen der Frühmoderne. Köln: SH, 1-20.

Marti, Hanspeter (2005): Kommunikationsnormen der Disputation. Die Universität Halle und Christian Thomasius als Paradigmen des Wandels. In: Ulrich Johannes Schneider (Hrsg.), Kultur der Kommunikation. Die europäische Gelehrtenrepublik im Zeitalter von Leibniz und Lessing. Wiesbaden: Harrassowitz, 317-344.

Marti, Hanspeter (2011): Dissertationen. In: Ulrich Rasche (Hrsg.), Quellen zur frühneuzeitlichen Universitätsgeschichte. Typen, Bestände, Forschungsperspektiven. Wiesbaden: Harrassowitz, 293-312.

Marti, Hanspeter (2014a): Altes festhalten, Neues suchen. Hallenser Disputationen im frühen 18. Jahrhundert. http://www.forschungen-engi.ch/projekte/halle.htm (25.10. 2016).

Marti, Hanspeter (2014b): Der Wettbewerb um die Gunst des Schicksals. Basler Kandidatenreigen um die Besetzung einer Rhetorikprofessur im 18. Jahrhundert. 
In: Aufklärung. Interdisziplinäres Jahrbuch zur Erforschung des 18. Jahrhunderts und seiner Wirkungsgeschichte 26, 237-257.

Marti, Hanspeter (2016a): Poetik, Rhetorik und Literaturkritik. Entstehung und Wirkung einer frühen Dissertation des Baslers Johann Bernhard Merian. In: Marion Gindhart, Hanspeter Marti \& Robert Seidel (Hrsg.), Frühneuzeitliche Disputationen. Polyvalente Produktionsapparate gelehrten Wissens, unter Mitarbeit von Karin Marti-Weissenbach. Köln u. a.: Böhlau, 255-284.

Marti, Hanspeter (2016b): Die frühneuzeitliche Schuldisputation. Stand, Perspektiven und Probleme ihrer Erforschung. In: Jan-Hendryk de Boer, Marian Füssel \& Jana Madlen Schütte (Hrsg.), Zwischen Konflikt und Kooperation. Praktiken der europäischen Gelehrtenkultur (12.-17. Jahrhundert). Berlin: Duncker \& Humblot, 281-305.

Marti, Hanspeter (2017): Frühneuzeitliches Disputationswesen und Fremdsprachen an der Universität Halle In: Max Häberlein \& Holger Zaunstöck (Hrsg.), Halle als Zentrum der Mehrsprachigkeit im langen 18. Jahrhundert. Halle: Franckesche Stiftungen, 15-35.

Marti-Weissenbach, Karin (2017): [Art.] Samuel Werenfels (Präses), Joachim Lydius \& Johann Georg Meyer (Respondenten), Dissertatio de meteoris orationis, Basel 1694. In: Hanspeter Marti, Reimund B. Sdzuj \& Robert Seidel (Hrsg.), Rhetorik, Poetik und Ästhetik im Bildungssystem des Alten Reiches. Wissenschaftshistorische Erschließung ausgewählter Dissertationen von Universitäten und Gymnasien 1500-1800. Köln u. a.: Böhlau, 228-243.

Merian, Johann Bernhard (Präses) \& Samuel Burckhardt (Respondent) (1743): Cogitationes quaedam de contemptu linguae Latinae. 23. April 1743. Basel: Johann Pistorius.

Merton, Robert K. (1980): Auf den Schultern von Riesen: ein Leitfaden durch das Labyrinth der Gelehrsamkeit, Frankfurt am Main: Syndikat.

Müller, Rainer A. (2003): Von der «Juristendominanz» zur «Medizinerschwemme». Zum Promotionswesen an deutschen Universitäten der Frühmoderne. In: Christian Hesse, Beat Immenhauser, Oliver Landolt \& Barbara Studer (Hrsg.), Personen der Geschichte Geschichte der Personen. Studien zur Kreuzzugs-, Sozial- und Bildungsgeschichte. Festschrift für Rainer Christoph Schwinges zum 60. Geburtstag. Basel: Schwabe, 317345.

Oexle, Otto Gerhard (2004): Begriff und Experiment. Überlegungen zum Verhältnis von Natur- und Geschichtswissenschaft. In: Vittoria Borsò, Christoph Kann (Hrsg.), Geschichtsdarstellung. Medien - Methoden - Setzungen. Köln u. a.: Böhlau, 19-56.

Paulsen, Friedrich (1896): Geschichte des gelehrten Unterrichts auf den deutschen Schulen und Universitäten vom Ausgang des Mittelalters bis zur Gegenwart. Mit besonderer Rücksicht auf den klassischen Unterricht. Erster Band. Leipzig: Veit \& Comp.

Poser, Hans (2001): Wissenschaftstheorie. Eine philosophische Einführung. Stuttgart: Reclam.

Pretzsch, Karl (1975): Verzeichnis der Breslauer Universitätsschriften 1811-1885. Hildesheim, New York: Olms.

Rother, Wolfgang (2003): Gelehrsamkeitskritik in der frühen Neuzeit. Samuel Werenfels' Dissertatio de logomachiis eruditorum und Idée d'un philosophe. In: Theologische Zeitschrift 59 (2), 137-159.

Rother, Wolfgang (2007): Paratus sum sententiam mutare: The influence of Cartesian Philosophy at Basle. In: History of Universities 22 (1), 73-97.

Schiewe, Jürgen (1996): Sprachenwechsel - Funktionswandel - Austausch der Denkstile. Die Universität Freiburg zwischen Latein und Deutsch. Tübingen: Max Niemeyer.

Schneider, Karl Ernst Christoph (1745): Diem natalem regis potentissimi et clementissimi Friderici Guilelmi IV. idibus octobr. h. XI. med. celebrandum mandato universitatis litterarum Vratislaviensis indicit C. E. Chr. Schneider, phil. Dr. P. O. Breslau: Universität. 
Steiner, Felix (2009): Dargestellte Autorschaft. Autorkonzept und Autorsubjekt in wissenschaftlichen Texten. Tübingen: Max Niemeyer.

Till, Dietmar (2004): Transformationen der Rhetorik. Untersuchungen zum Wandel der Rhetoriktheorie im 17. und 18. Jahrhundert. Tübingen: Max Niemeyer.

Verzeichniß der Vorlesungen (1874), welche im Winterhalbjahre 1874-75 an der Königlichen Universität Greifswald und an der Königlichen staats= und landwirthschaftlichen Akademie Eldena gehalten werden sollen. Greifswald: F. W. Kunicke.

Vom Bruch, Rüdiger (2001): Die Gründung der Berliner Universität. In: Rainer Christoph Schwinges (Hrsg.), Humboldt International. Der Export des deutschen Universitätsmodells im 19. und 20. Jahrhundert. Basel: Schwabe, 53-73.

Werenfels, Samuel (1739): Dissertatio de logomachiis eruditorum. In: Samuel Werenfels, Opuscula theologica, philosophica et philologica. Editio altera ab auctore recognita, emendata et aucta. Tomus secundus. Lausanne, Genf: Bousquet, 1-116. 